\title{
Understanding the light curves of the HST-1 knot in M87
}

\section{Y. U. Coronado and S. Mendoza}

Instituto de Astronomia, Universidad Nacional Autonoma de Mexico.

E-mail: coronado@astro.unam.mx, sergio@astro.unam.mx

The motion of knots in astrophysical jets is commonly interpreted as shock waves moving along it. Observations of the HST-1 knot during extended periods of time have produced complicated light curves on many wavelengths which are difficult to account using standard hydrodynamical models. Here we reproduce these light curves using the semi-analytical approach developed by Mendoza et al. (2009), developed to reproduce light curves of working surfaces moving along relativistic jets. These working surfaces are generated by periodic oscillations of the injected flow velocity and discharge at the base of the jet. In particular, we use this approach to reproduce the exotic observed features of the light curves of the HST-1 knot in M87. We show that the complicated fits to the light curves are reproduced with high accuracy in all wavelengths. As a bonus, we show that this model is also able to reproduce the light curve of the micro-qsr A0620000 with high accuracy.

25th Texas Symposium on Relativistic Astrophysics - TEXAS 2010

December 06-10, 2010

Heidelberg, Germany 


\section{Introduction}

Chandra Observations of the HST-1 knot [1] in M87 showed that a peak in the X-ray light curve developed about 2005. This light curve has since shown successive peaks over short periods of time. Observations in UV [2] and radio [3] have also shown the same trend. Since quasiperiodic signatures in the brightening and dimming of the HST-1 knot X-ray observations were found [4], this shows a manifestation of a previous modulation in the jet power, most probably a local oscillation of the process that converts the bulk kinetic jet power to internal energy of the emitting plasma.

The key to understand the particular features in the HST-1 knot of M87 is the physical basis behind its light curve. To give an idea of the complexities observed, there has been a discussion about the X-ray observations as to whether the main contribution in this wavelength is an effect of the hot accretion disc with its corona [5] or a particular feature of some kind of recollimation shock [6]. We mention here that radio observations show that the knot is well isolated from the nucleus since the activity is displaced away from the central engine by $\gtrsim 120 \mathrm{pc}$. Also, these radio observations show superluminal motion of the knot, which means that its bulk velocity is highly relativistic.

In this article we take multi-wavelength observations from different sources. For instance, the X-rays observations are part of a multi-frequency program coordinating the Chandra and HST monitoring by [4]. The ultraviolet data are part of the observations carried out during the years 1999 to 2006 [2]. Finally the radio data corresponds to observations with the VLBI at $2 \mathrm{~cm}$ [3]. All these observations reveal a clear multi frequency light curve of the HST-1 knot which serves as a laboratory to test the ideas developed by [7] for the formation and propagation of the working surface of a relativistic jet, with periodic variations of the injected velocity profiles and mass rate outflows.

\section{Model}

The formation of internal shock waves on a relativistic jet are commonly explained by different mechanisms, such as the interaction of the jet with inhomogeneities of the surrounding medium, the bending of jets and time fluctuations in the parameters of the ejection $[8-10,7]$. Here we are concerned with the latter. When the speed of the emitted mass particles varies with time, a faster but later fluid parcel eventually hits an earlier but slower ejection producing an initial discontinuity which gives rise to a working surface, i.e. a hydrodynamical object formed by two shock waves separated by a contact surface. In the frame of reference of the central engine, where the jet is being ejected, the working surface travels along the jet with an average velocity $v_{\mathrm{ws}}$. (see e.g. [8]). In what follows we use the relativistic semi-analytical model of [7] to describe a working surface and its kinetic luminosity power travelling inside an astrophysical jet. To do this, we consider a source ejecting material in a preferred direction $x$ with a velocity $v(\tau)$ and a mass ejection rate $\dot{m}(\tau)$, both dependent on time $\tau$ as measured from the jet's source [7]. The energy loss inside the working surface is calculated as the difference between the initial energy of the material, when it was injected at the base of the jet, and the energy of the flow inside the working surface. Assuming an efficient mechanism which converts all this kinetic energy into radiation power, then the total 
luminosity is given by $L:=\mathrm{d} E_{\mathrm{r}} / \mathrm{d} t$, where $E_{\mathrm{r}}=E_{0}-E_{\mathrm{ws}}$ is the radiated energy within the working surface [7].

In what follows we use the approach followed by [7] in order to show that their model can describe the important features observed on the evolution of $H S T-1$. To do so, the injected velocity is assumed to have a periodic variation given by $v(\tau)=v_{0}+c \eta^{2} \sin \omega \tau$, with a constant mass discharge $\dot{m}$ and a velocity of light $c$. We mention here that in order to perform the computations it is best to numerically assume a dimensionless system of units for which the oscillating frequency $\omega=1$ and $\dot{m}=1$. We have used this approach, which is also followed by [7], but we will present our results on the physical system of units for which $\omega$ and $\dot{m}$ are dimensional constants.

\section{Numerical fits}

The data sample of HST-1 covers a period of time between the years 2000 to 2009. Since we are going to use observations on different wavelengths, then it is best to normalise all observations to the intrinsic luminosity of HST-1. To do so, for X-rays we use the procedure developed in [1], which gives a power law index for the flux of 1.5 at a wavelength of $2 \mathrm{~cm} \mathrm{[3].} \mathrm{For} \mathrm{the} \mathrm{UV} \mathrm{data} \mathrm{we}$ use the flux density using the reference wavelength of the camera ACS/F220W at 2255.5AA [2].

Since the correction in flux is given, we can now use the standard relation between luminosity and flux without worrying about extinction. To do so, we assume an isotropic emission of the source at a distance of $16 \mathrm{Mpc}$ [11], which gives a lower limit in the luminosity emitted by the HST-1 knot.

On the other hand, the mean velocity $v_{0}$ of the jet is taken as $v_{0}=0.98 c$ which is in agreement with the observed value of a Lorentz Factor $\gamma=6$ [12]. The value of the parameter $\eta^{2}$ and frequency are selected by linear fits in light curve of the observed data.

The X-ray light curve does not work with a simple variation of the velocity and so, we additionally adopt a periodic variation on the injected mass given by $\dot{m}=\dot{m}_{i}+\psi \sin \Omega$ just for the first flare. After the peak, the standard assumption made above fits quite well the observations.

In all the observed light curves, there is at least one subsequent increase in luminosity after the maximum peak. These local peaks can be easily modelled by assuming a rapid variation on the value of the discharge $\dot{m}$ injected in the jet according to the high variability of the core of M87 [4].

Figures 1-3 show the obtained fits, which are in good agreement with the observations. However, all of these take into account the bolometric luminosity of the flow. We have calculated in Figure 4 the variation of the spectral index as a function of time to fit our model, with data obtained by [1]. From the figure it follows that the X-ray luminosity is dominant at all times.

\section{Discussion}

We have described the light curve of the knot in HST-1 on different wavelengths using the relativistic model of the evolution of working surfaces moving along a jet by [7]. The physical mechanism responsible of the emission is still not known since, as described by [1], a simple synchrotron emission can't be accounted due to a break in the main burst. 


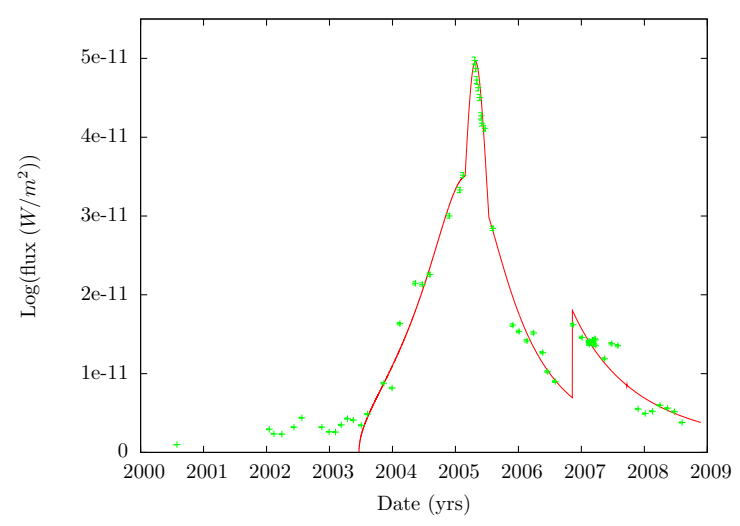

Figure 1: The figure shows as points the observed light curve in X-Ray of HST-1 from [4]. The continuous curve on the plot is the best fit using the semi-analytical model described in the text with a linear fit to the data yielding $\dot{m}=5.59 \times 10^{-7} M_{\odot} / y r$ and $\omega=0.1207$. The second peak after the maximum is modelled as an increase in the discharge of $\dot{m}=1.10 \times 10^{-7} M_{\odot} / y r$ at time 2006.86 with a duration of 1.64 years.

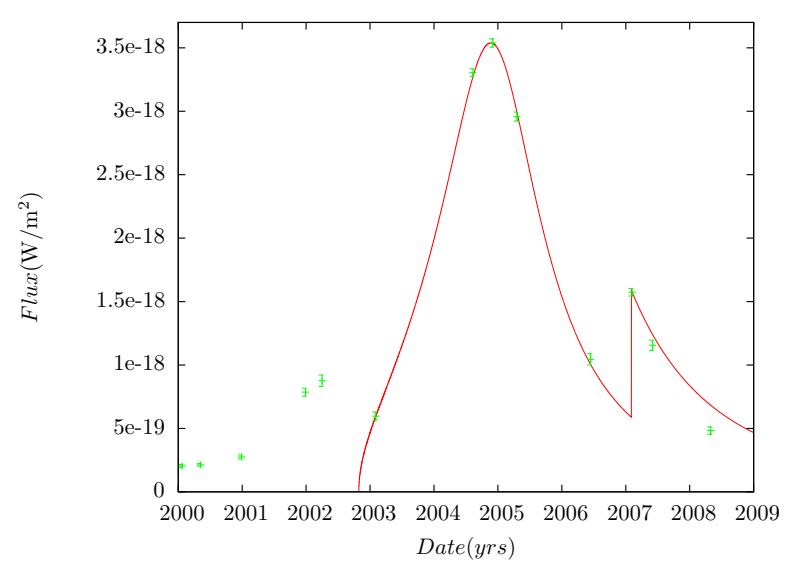

Figure 3: The figure shows the observed points of the HST-1 radio light curve from the observations of [3]. The continuous curve on the figure is the best fit to the observations using the semi-analytical model described in the text with a linear fit to the data yielding $\dot{m}=2.11 \times 10^{-11} M_{\odot} / y r$ and $\omega=1.0$. The second peak after the maximum is modelled as an increase in the discharge of $\dot{m}=3.48 \times 10^{-12} M_{\odot} / y r$ at time 2007.1 with a duration of 2.0 years.

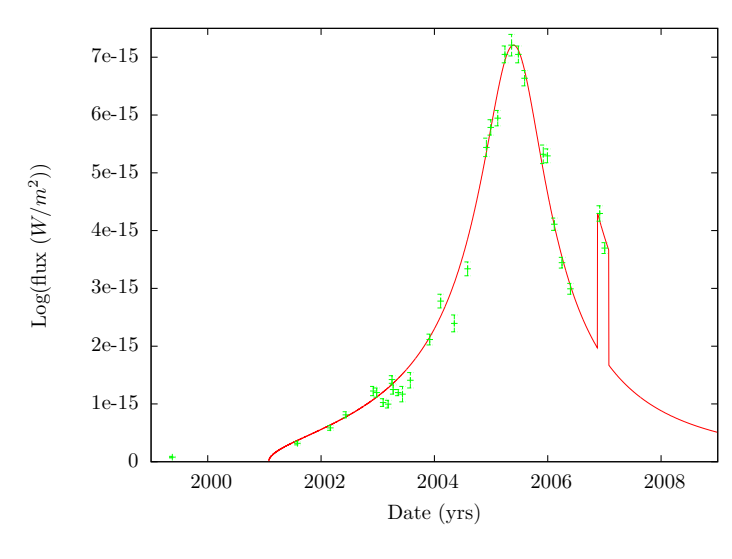

Figure 2: The figure shows the observed points of the HST-1 UV light curve from [2]. The continuous curve on the graph is the best fit to the observations using the semi-analytical model described in the text with a linear fit to the data yielding $\dot{m}=6.52 \times 10^{-6} M_{\odot} / y r$ and $\omega=0.02528$. The second peak after the maximum is modelled as an increase in the discharge of $\dot{m}=1.78 \times 10^{-6} M_{\odot} / y r$ at time 2006.88 with a duration of 0.2 years.

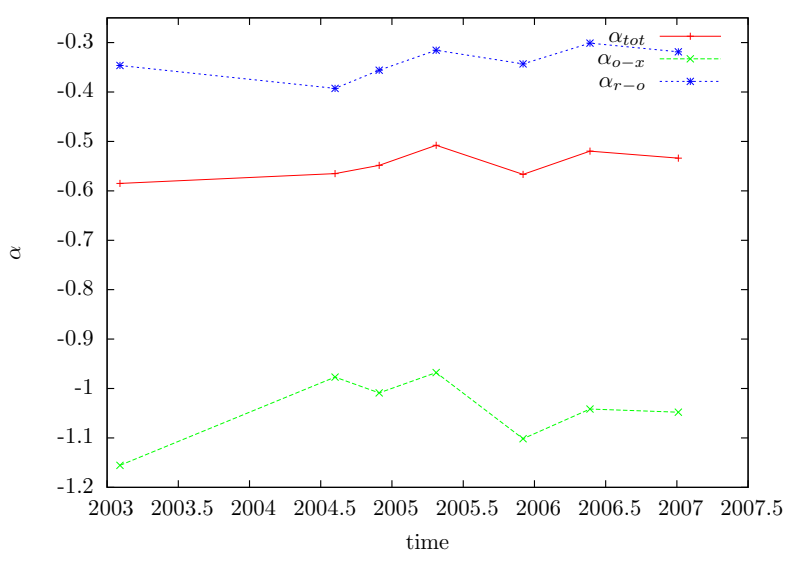

Figure 4: The figure show the time $t$ evolution of the spectral index $\alpha$. The upper and lower curves are the spectral values from radio to UV ( $2 \mathrm{cmto} 225.5 \mathrm{~nm}$ ) and from UV to X.rays $(225.5 \mathrm{~nm}$ to $2 \mathrm{KeV})$ respectively. 


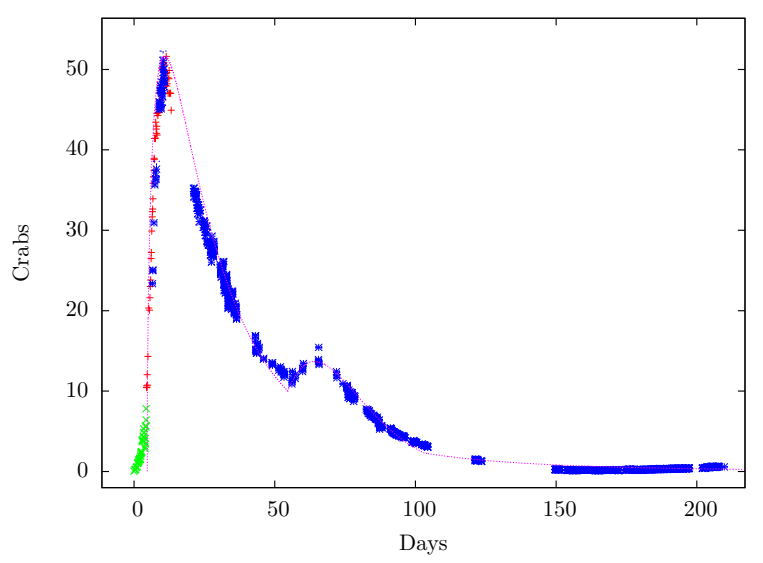

Figure 5: The figure show a burst on the X-ray light curve of the microquasar A06200-00. The continuous curve on the plot is the best fit using the semi-analytical model described in the text with a linear fit to the data yielding $\dot{m}=3.27 \times 10^{-17} M_{\odot} / y r$ and $\omega=1.0$. The second peak after the maximum is modelled as an increase in the discharge of $\dot{m}=7.2 \times 10^{-14} M_{\odot} / y r$ at time 55 with a duration of 45 days.

To see the power of the semi-analytic model of [7], we also adopt the same procedure in Figure 5 but for the $\mu$-quasar A0620-00 which has been observed in detail for quite a nice period of time.

Since the same model has been used to fit light curves of long gamma-ray bursts by [7], all this means that light curves that produce bursts can be easily modelled by using this approach and that certainly the burst are simply two shock waves forming due to the variations of the injected flow.

\section{Acknowledgments}

This work was supported by a DGAPA-UNAM grant (PAPIIT IN116210-3). The authors acknowledge support from CONACyT $(210965,26344)$ and thank J. McClintock for providing the observations of the $\mu$-qsr A0620-00.

\section{References}

[1] D. E. Harris, C. C. Cheung, J. A. Biretta, W. B. Sparks, W. Junor, E. S. Perlman, and A. S. Wilson. The Outburst of HST-1 in the M87 Jet. ApJ, 640:211-218, Mar. 2006.

[2] J. P. Madrid. Hubble Space Telescope Observations of an Extraordinary Flare in the M87 Jet. Astronomical Journal, 137:3864-3868, Apr. 2009.

[3] C. S. Chang, E. Ros, Y. Y. Kovalev, and M. L. Lister. VLBI detection of the HST-1 feature in the M 87 jet at $2 \mathrm{~cm}$. åp, 515:A38+, June 2010.

[4] D. E. Harris, C. C. Cheung, Ł. Stawarz, J. A. Biretta, and E. S. Perlman. Variability Timescales in the M87 Jet: Signatures of $E^{2}$ Losses, Discovery of a Quasi Period in HST-1, and the Site of TeV Flaring. ApJ, 699:305-314, July 2009.

[5] C. C. Cheung, D. E. Harris, and Ł. Stawarz. Superluminal Radio Features in the M87 Jet and the Site of Flaring TeV Gamma-Ray Emission. ApJL, 663:L65-L68, July 2007. 
[6] Ł. Stawarz, F. Aharonian, J. Kataoka, M. Ostrowski, A. Siemiginowska, and M. Sikora. Dynamics and high-energy emission of the flaring HST-1 knot in the M 87 jet. MNRAS, 370:981-992, Aug. 2006.

[7] S. Mendoza, J. C. Hidalgo, D. Olvera, and J. I. Cabrera. Internal shocks in relativistic jets with time-dependent sources. MNRAS, 395:1403-1408, May 2009.

[8] M. J. Rees and P. Meszaros. Unsteady outflow models for cosmological gamma-ray bursts. ApJL, 430:L93-L96, Aug. 1994.

[9] S. Mendoza. Shocks and Jets in Radio Galaxies and Quasars. PhD thesis, Cavendish Laboratory, Cambridge University U.K., available at

http: //www.mendozza.org/sergio/phdthesis, 2000.

[10] O. Jamil, R. P. Fender, and C. R. Kaiser. Internal Shocks Model for Microquasar Jets. In Microquasars and Beyond, 2008.

[11] A. Jordán, P. Côté, J. P. Blakeslee, L. Ferrarese, D. E. McLaughlin, S. Mei, E. W. Peng, J. L. Tonry, D. Merritt, M. Milosavljević, C. L. Sarazin, G. R. Sivakoff, and M. J. West. The ACS Virgo Cluster Survey. X. Half-Light Radii of Globular Clusters in Early-Type Galaxies: Environmental Dependencies and a Standard Ruler for Distance Estimation. ApJ, 634:1002-1019, Dec. 2005.

[12] J. A. Biretta, W. B. Sparks, and F. Macchetto. Hubble Space Telescope Observations of Superluminal Motion in the M87 Jet. ApJ, 520:621-626, Aug. 1999. 